\title{
Combination treatment with cisplatin, paclitaxel and olaparib has synergistic and dose reduction potential in ovarian cancer cells
}

\author{
JIANWEN GAO ${ }^{1-3^{*}}$, ZEHUA WANG $^{4,5^{*}}$, JIAYU FU $^{1}$, JISAIHAN A. $^{1}$, YUKO OHNO $^{1}$ and CONGJIAN XU ${ }^{4-6}$ \\ ${ }^{1}$ Department of Mathematical Health Science, Graduate School of Medicine, Osaka University, Osaka 565-0871, Japan; \\ ${ }^{2}$ School of Medical Engineering, Ma'anshan University, Ma'anshan, Anhui 243100; \\ ${ }^{3}$ Department of Biotechnological Pharmaceutics, Shanghai Pharmaceutical School, Shanghai 200135; \\ ${ }^{4}$ Obstetrics and Gynecology Hospital of Fudan University; ${ }^{5}$ Shanghai Key Laboratory of \\ Female Reproductive Endocrine Related Diseases, Shanghai 200011; ${ }^{6}$ Department of Obstetrics and Gynecology of \\ Shanghai Medical School, Fudan University, Shanghai 200032, P.R. China
}

Received March 30, 2020; Accepted September 29, 2020

DOI: $10.3892 / \mathrm{etm} .2021 .10367$

\begin{abstract}
Ovarian cancer is the most lethal type of gynecological cancer. Due to its high heterogeneity and complicated pathological mechanisms, the 5-year survival rate of patients with ovarian cancer is $<40 \%$. Tumor cytoreductive surgery and systemic chemotherapy of platinum combined with paclitaxel are currently considered the gold standard for the treatment of ovarian cancer, and chemotherapy resistance has become a key constraint in improving the cure rate of ovarian cancer. Therefore, it is important to identify novel treatment methods and strategies for ovarian cancer. Targeted drugs can not only be used in combination with chemotherapy, but also act as maintenance therapy to promote patient survival time. PARP inhibitor is a novel type of ovarian cancer treatment targeted drug, which can induce an anticancer effect by inhibiting DNA damage and repair of ovarian cancer cells. The present study investigated the different effects of olaparib, cisplatin and paclitaxel in several dosages by single use and combinations on the proliferation of different human ovarian cancer cell lines, in order to verify the synergistic effects of the combinations of the three anticancer agents in pairs. The proliferation inhibitory rate of the cell lines was determined using a Cell Counting Kit- 8 assay, while the combination index (CI) value of the combination of three agents in pairs was analyzed using Compusyn software. The proliferation was observed using a crystal violet assay, and the apoptosis ratio was measured via flow cytometry. The results
\end{abstract}

Correspondence to: Professor Yuko Ohno, Department of Mathematical Health Science, Graduate School of Medicine, Osaka University, 1-7 Yamadaoka, Suita, Osaka 565-0871, Japan

E-mail: 25b18822@sahs.med.osaka-u.ac.jp

Professor Congjian Xu, Obstetrics and Gynecology Hospital of Fudan University, 419 Fangxie Road, Shanghai 200011, P.R. China

E-mail: xucongjian@fudan.edu.cn

${ }^{*}$ Contributed equally

Key words: olaparib, cisplatin, paclitaxel, ovarian cancer of the present study revealed that the combination of cisplatin with olaparib group had a higher inhibition effect than each single group and had a higher dose-reduction index of $>1$ than the other two combinations at all concentrations in A2780 and OVCAR-3 cell lines. The difference in proliferation inhibition and induced apoptosis rate of A2780 cell lines was significant in the combination of cisplatin with olaparib group and the control group $(\mathrm{P}<0.01)$ at $0.25 \mathrm{x} \mathrm{IC}_{50}$. For the OVCAR-3 cell line, the difference was also significant between two groups $(\mathrm{P}<0.05)$. The CI values in the A2780 cell line revealed significant differences between the low-dose group $(0.0625 \mathrm{x}, 0.125 \mathrm{x}$ and $0.25 \mathrm{x}$ $\left.\mathrm{IC}_{50}\right)$ and the high-dose group (0.5x, 1.0x and 2.0x $\left.\mathrm{IC}_{50}\right)$ for the group that received the combination of cisplatin with olaparib $(\mathrm{P}<0.05)$. The present study highlighted that the group receiving a combination of cisplatin with olaparib exhibited the most significant synergistic effects among the three combinations, particularly at low doses.

\section{Introduction}

Ovarian cancer exhibits the highest mortality of all gynecological malignancies, due to the non-specific symptoms and insufficient diagnostic approaches. The majority of patients do not know they have the disease until they receive a diagnosis at an advanced stage. Cytoreductive surgery plus post-operative adjunctive chemotherapy is currently considered the standard protocol for treatment. Due to the undesirable efforts of the major administration on late-stage ovarian cancer, feasible chemotherapy after surgery is of high importance $(1,2)$. As a cell cycle non-specific agent, cisplatin suppresses the DNA duplication and transcription by binding intracellular DNA into a cisplatin-DNA adduct or complexing with cytoplasmic proteins and nucleoproteins. Accordingly, cisplatin has been adopted in chemotherapy for numerous types of cancer (e.g., ovarian cancer, gastric cancer and bladder cancer) (3). Paclitaxel refers to one type of antimicrotubular agent, intracellularly binding to microvascular proteins with the effect of facilitating polymerization, and thwarts normal microvascular depolymerization. Thus, the agent can regulate microvascular homeostasis in the body, thwart the mitosis and proliferation of cancer cells, 
and predominate in the treatment of breast cancer and ovarian cancer (4). According to several years of clinical practice, the strategy of paclitaxel plus cisplatin has enhanced the clinical efficacy of ovarian cancer. Despite enormous efforts to improve surgical treatment and combination chemotherapy for the disease, the 5-year survival rate remains to be $25-35 \%$ (5).

As the studies on the pathogenesis of ovarian cancer have been deepening, molecular targeted therapy for cancer continues to make novel achievements. Targeted drugs exert specific killing effects on tumor cells based on single drug, combined chemotherapeutic agents or combined targeted drugs; they have been adopted as a novel strategy for ovarian cancer therapy. Poly ADP-ribose polymerase (PARP), a DNA repair enzyme, exists in most eukaryotic cells, and it causes cleavage of caspase, a core member in apoptosis. By recognizing the impaired structure of DNA pieces, PARP can be activated to mitigate DNA damage signaling pathways. It has been commonly considered a sensor of DNA damage and to be critical in DNA damage repair $(6,7)$. Recently, studies have suggested that the most acceptable mechanisms underlying DNA damage repair include base excision repair (BER), nucleotide excision repair (NER), mismatch repair (MMR), as well as homologous recombination (HR). To be specific, PARP largely predominates the repair of single-strand breaks (SSBs) of BER (8). When PARP is devoid, or its inhibitory activity occurs, i.e., functional defects in BER, the resulting irreparable SSBs will form double-strand breaks (DSBs) due to the decreased replication forks. Nevertheless, the continuously accumulated DSBs become irreparable by HR, and the consequent cytotoxicity leads to synthetic lethality, killing targeted tumor cells and exerting anti-tumor effects. The PARP family consists of 17 members with PARP-1/2 as the existing research emphasis (9). Poly ADP-ribosylation (PARylation), catalyzed by the two enzymes participating in DNA damage repair, is vital to the pathogenesis and development of multiple types of tumor $(10,11)$. BER is critical to restore SSBs by PARP-1/2. However, it is the restoration that develops radiotherapy or chemotherapy resistance (e.g., alkylating agents) $(12,13)$. Numerous studies have suggested that the overexpression of PARP in tumor cells may induce therapy resistance. Since the suppression of PARP can decrease anti-tumor drug resistance, PARP has been highlighted as a novel target for cancer therapy (14).

PARP inhibitor as a novel targeted agent for ovarian cancer is a potential option for targeted therapy, by selectively inhibiting PARP. Numerous studies have revealed that PARP inhibitors have favorable anti-tumor effects on recurrent ovarian cancer with a good drug tolerance, significantly prolonging the progression-free survival (PFS) of patients with the BRCA gene, i.e., a susceptibility gene of breast cancer (15). Olaparib is a newly approved peroral PARP inhibitor, having been prioritized in a review from European Medicines Agency (EMA) and Food and Drug Administration (FDA) in sequence (16). It has been approved in Europe and the USA on December 18 and 19, 2014, respectively (17). Another study demonstrated that olaparib, a PARP inhibitor, has anti-tumor effects on high-grade-serous ovarian cancer, and is closely associated with platinum sensitivity (18). The combination of olaparib and other chemotherapeutic agents also exhibits significant anti-tumor effects, with basic drug tolerance $(19,20)$. A recent study reported that treatment with olaparib improves the PFS of patients with epithelial ovarian cancer that possess a non-mutated BRCA gene (21). These results indicated that the patients with homologous recombination deficits also potentially respond to PARP inhibitors, except for those that possess a mutated BRCA gene. The present study aimed to determine the effects of single or pairwise combinations of olaparib, cisplatin and paclitaxel on ovarian cancer cell lines, with the hope of providing a novel strategy for its treatment in the clinical setting.

\section{Materials and methods}

Cell lines and culture conditions. The A2780 and OVCAR-3 human epithelial ovarian cancer cell lines were obtained from the American Type Culture Collection. A2780 cells were cultured in a humidified $5 \% \mathrm{CO}_{2}$ incubator at $37^{\circ} \mathrm{C}$ with RPMI-1640 medium supplemented with $10 \%$ fetal bovine serum (Gibco, Invitrogen) and $1 \%$ penicillin/streptomycin solution. OVCAR-3 cells were cultured in a humidified $5 \% \mathrm{CO}_{2}$ incubator at $37^{\circ} \mathrm{C}$ with RPMI-1640 medium (Gibco; Invitrogen) supplemented with $20 \%$ fetal bovine serum and $1 \%$ penicillin/streptomycin solution. The medium was replaced every other day. The cells were detached with $0.25 \%$ tyrosinase and passaged when the cells reached $75-85 \%$ confluency.

Drug treatments. Olaparib, cisplatin and paclitaxel were purchased from Selleck Chemicals and prepared in $10 \mathrm{mM}$ stocks. The initial concentrations of olaparib, cisplatin and paclitaxel were $160 \mu \mathrm{M}, 800 \mu \mathrm{M}$ and $160 \mathrm{nM}$, respectively, for the half maximal inhibitory concentration $\left(\mathrm{IC}_{50}\right)$ determinations. Agents were diluted to 7 concentrations with 4-fold serial dilutions. After the $\mathrm{IC}_{50}$ values were determined, concentrations of three agents were set to $0.0625 \mathrm{x}, 0.125 \mathrm{x}$, $0.25 \mathrm{x}, 0.5 \mathrm{x}, 1.0 \mathrm{x}$ and $2.0 \mathrm{x} \mathrm{IC}_{50}$ in the subsequent drug combination experiments.

Proliferation inhibitory rate via cell counting kit-8 (CCK-8) assay. The CCK-8 assay (Dojindo Molecular Technologies, Inc.) was utilized for the detection of proliferation inhibitory rate. A2780 and OVCAR-3 cells were treated by diluted agents (Gibco, Invitrogen) in 96-well culture plates at $5 \times 10^{3}$ cells per well for $48 \mathrm{~h}$. After $2 \mathrm{~h}$ incubation with the CCK-8 reagent (10 $\mu \mathrm{l} /$ well) at $37^{\circ} \mathrm{C}$, the $\mathrm{OD}_{450}$ values of plate wells were recorded with a microplate reader. The proliferation inhibitory rates of A2780 and OVCAR-3 cells were ascertained using the following equation: Proliferation inhibitory rate $=\left[\left(1-\left(\mathrm{OD}_{\text {treatment }}\right.\right.\right.$ group $\left.\left.-\mathrm{OD}_{\text {intact group }}\right) /\left(\mathrm{OD}_{\text {control group }}-\mathrm{OD}_{\text {intact group }}\right)\right] \times 100 \%$. Each group had 3 readings, and the experiment was performed in triplicate.

Crystal violet staining. Cells were inoculated in $60 \mathrm{~mm}$ cell culture dishes at a density of $1 \times 10^{5}$ cells per dish in triplicate. The concentration of cisplatin and olaparib were administered as $0.25 \mathrm{x} \mathrm{IC}$ 50 for both single and combination use. The cells were cultured for $48 \mathrm{~h}$ before being stained with crystal violet at room temperature. Images of cells were captured with a fluorescence microscope at x100 magnification, and the area fraction of the cell staining was analyzed by ImageJ 2.0 software.

Flow cytometry detection. A2780 and OVCAR-3 cells were inoculated into 6-well plates. When the cell confluency reached 
A

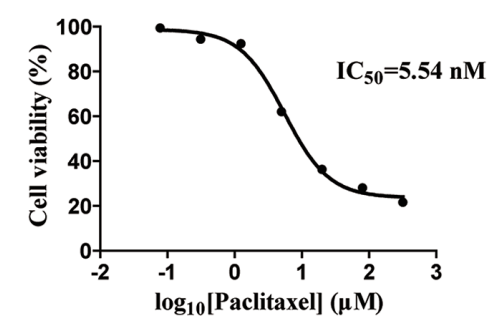

B

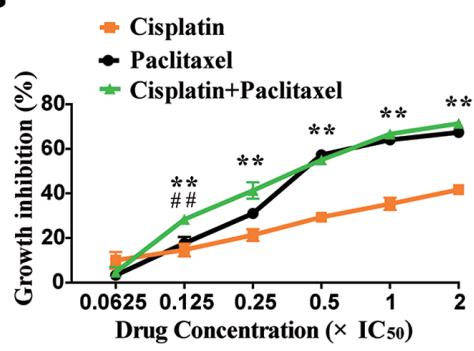

C

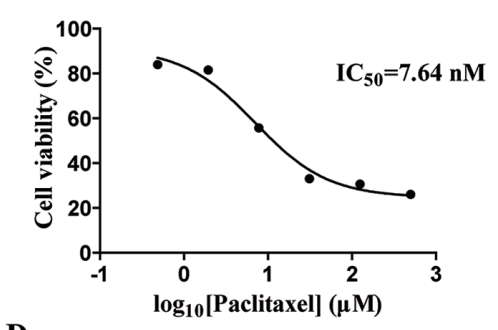

D

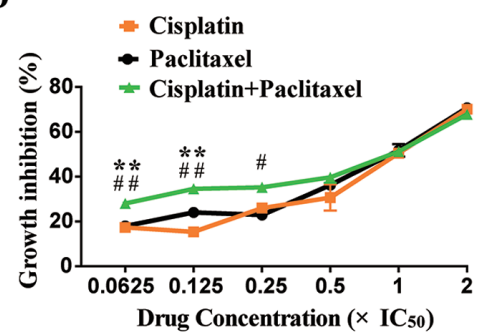

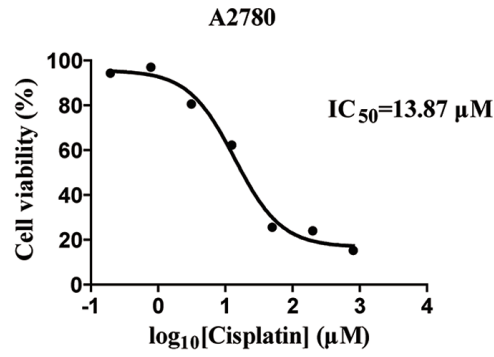
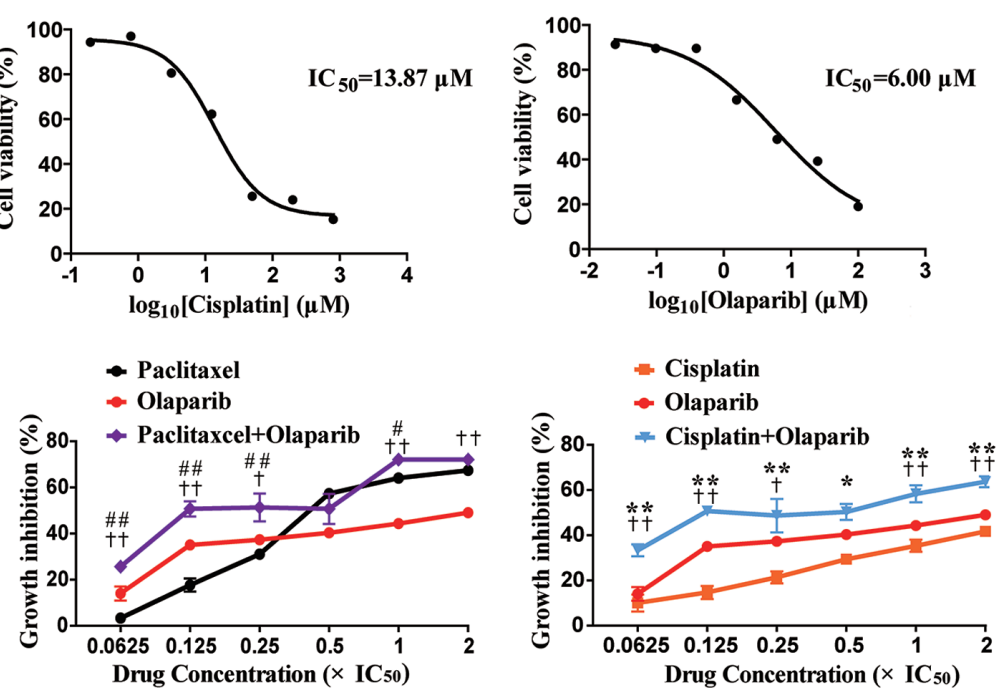

OVCAR-3
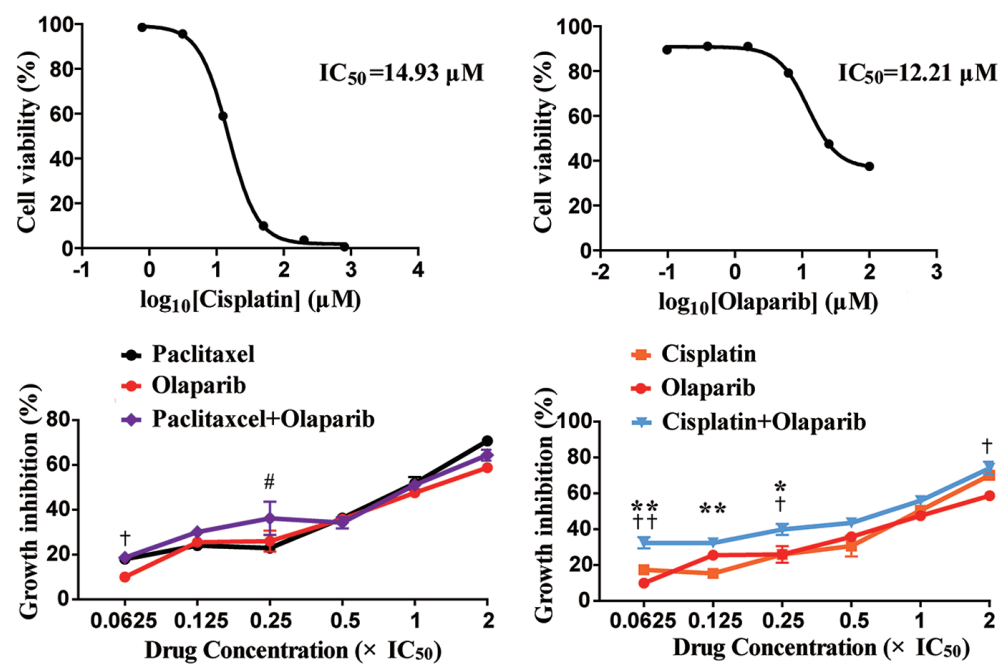

Figure 1. Growth inhibitory effects of single and combined anticancer agents on A2780 and OVCAR-3 cells. (A) $\mathrm{IC}_{50}$ values determination of three single agents in A2780 cells. (B) Growth inhibitory rates of A2780 cells treated with the combination of three agents in pairs. (C) $\mathrm{IC}_{50}$ values determination of three single agents in OVCAR-3 cells. (D) Growth inhibitory rates of OVCAR-3 cells with the combination of three agents in pairs. "P $\mathrm{P}<0.05$ and ${ }^{* *} \mathrm{P}<0.01$, combination group vs. cisplatin group; ${ }^{\dagger} \mathrm{P}<0.05$ and ${ }^{\dagger} \mathrm{P}<0.01$, combination group vs. olaparib group; ${ }^{\sharp} \mathrm{P}<0.05$ and ${ }^{\# \#} \mathrm{P}<0.01$, combination group vs. paclitaxel group.

$60-70 \%$, cisplatin, olaparib, cisplatin + paclitaxel, paclitaxel + olaparib, cisplatin + olaparib, and the medium set as the control group were added at a density of $1 \times 10^{5}$ cells per well in triplicate. Cisplatin, paclitaxel and olaparib were administered at $0.25 \mathrm{x} \mathrm{IC}_{50}$ for both single and combination use. After $48 \mathrm{~h}$ incubation, cells in all groups were collected and then centrifuged at $1,000 \mathrm{rpm}$ for $3 \mathrm{~min}$ at $4^{\circ} \mathrm{C}$; next, the supernatant was removed, and $1 \mathrm{ml}$ PBS was added for resuspension, and the cells were recentrifuged at $1,000 \mathrm{rpm}$ for $3 \mathrm{~min}$ at $4^{\circ} \mathrm{C}$. The selected cells were stained using an Annexin V-FITC apoptosis kit (Dojindo), and cell apoptosis was determined via flow cytometry. FITC and PI fluorescence were detected by 525 and $620 \mathrm{~nm}$ bandpass filters excited at the wavelength of $488 \mathrm{~nm}$, and fluorescence signals of 20,000 cells were collected in each sample.

Compusyn software analysis. Compusyn software (ComboSyn, Inc.) analysis was used to assess the synergistic, additive and antagonistic effects of the anti-cancer agents.
The Chou-Talalay method (22) for drug combination was employed to quantitatively determine the interactions between two agents. The combination index (CI) value was based on the multiple drug-effect equation and it could be calculated by $\mathrm{CI}=\left(\mathrm{D}_{1} / \mathrm{D}_{\mathrm{X} 1}\right)+\left(\mathrm{D}_{2} / \mathrm{D}_{\mathrm{X} 2}\right) \cdot \mathrm{D}_{1}$ and $\mathrm{D}_{2}$ denote the doses of agents 1 and 2 respectively, as the combination reached a certain proliferation inhibitory rate, while $\mathrm{D}_{\mathrm{X} 1}$ and $\mathrm{D}_{\mathrm{X} 2}$ denote the doses of single agents 1 and 2 under that proliferation inhibitory rate. $\mathrm{CI}<1$ is considered to indicate synergistic effects of the two combined agents on inhibition of tumor cell proliferation, while $\mathrm{CI}>1$ was considered an antagonistic effect.

Statistical analysis. Each experiment was performed in triplicate. All data were analyzed using SPSS software (version 20.0). Student's t-test was used for comparisons between two groups. The differences among multiple groups were analyzed via one-way ANOVA with Tukey's post hoc test. $\mathrm{P}<0.05$ was considered to indicate a statistically significant difference. 
A
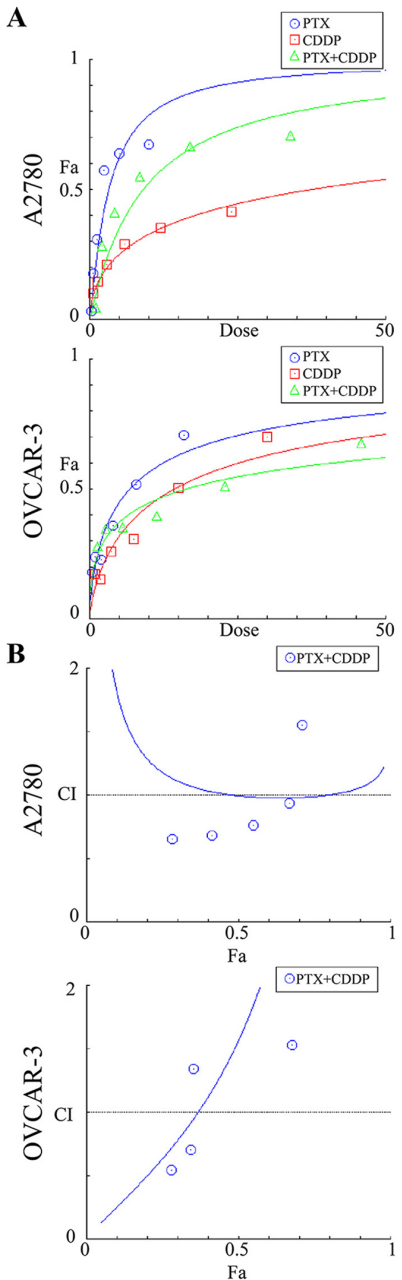

C
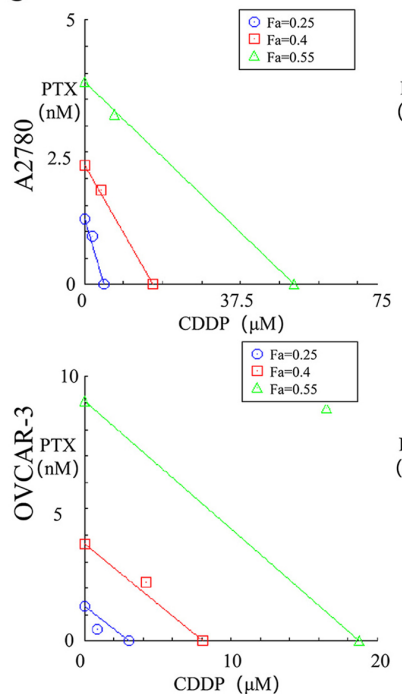
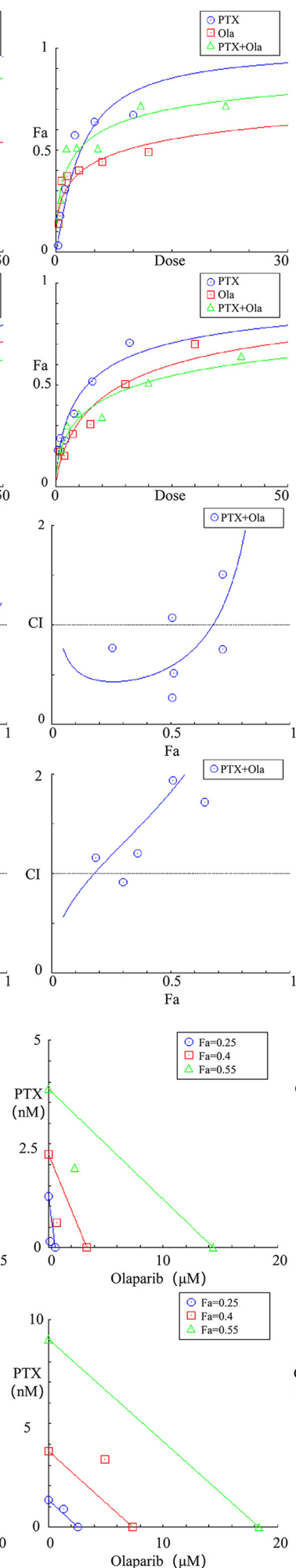
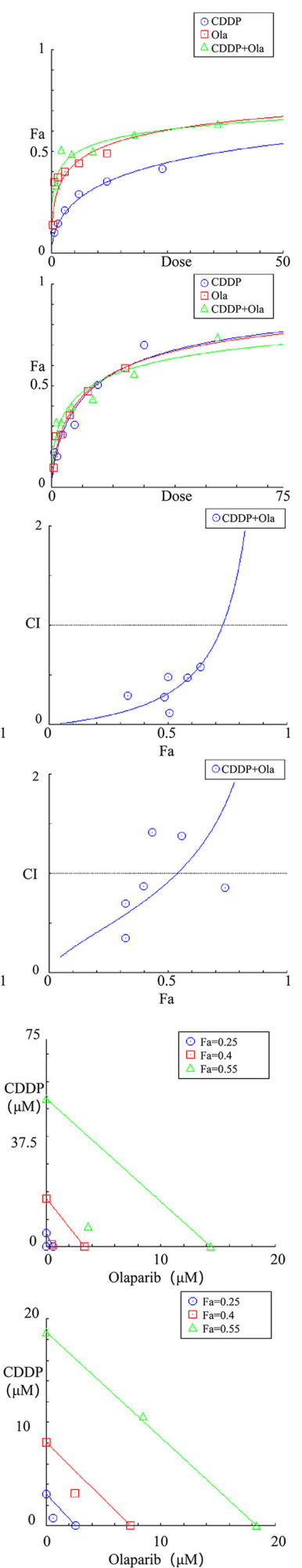

Figure 2. Graphic representations obtained from the CompuSyn report for combinations of CDDP, PTX and Ola in pairs. (A) Dose-effect curves. (B) CI plot. (C) Polygonogram at Fa=0.25. CDDP, cisplatin; $\mathrm{CI}$, combination index; Fa, fractional inhibition; Ola, olaparib; PTX, paclitaxel.

\section{Results}

Proliferation inhibitory effects of single and combined anti-cancer agents on A2780 and OVCAR-3 cells. In the present study, the $\mathrm{IC}_{50}$ values of cisplatin, paclitaxel and olaparib were determined via a CCK-8 assay. For the A2780 cell line, the $\mathrm{IC}_{50}$ values of cisplatin, paclitaxel and olaparib cell lines were $13.87 \pm 0.08,5.54 \pm 0.21$ and $6.00 \pm 0.35$ (SEM) $\mu \mathrm{M}$, respectively. For the OVCAR-3 cell line, the $\mathrm{IC}_{50}$ values of cisplatin, paclitaxel and olaparib were $14.93 \pm 0.07,7.64 \pm 0.14$ and $12.21 \pm 0.10$ (SEM) $\mu \mathrm{M}$, respectively (Fig. $1 \mathrm{~A}$ and C).

To assess the inhibitory effects of the single or combined use of the agents on the proliferation of A2780 and OVCAR-3 cells, three groups were set as two single agent groups and one 
combination agent group. The proliferation inhibitory rates of the A2780 and OVCAR-3 cells at 6 concentrations $(0.0625 \mathrm{x}$, $0.125 \mathrm{x}, 0.25 \mathrm{x}, 0.5 \mathrm{x}, 1.0 \mathrm{x}$ and $2.0 \mathrm{x} \mathrm{IC}_{50}$ ) were ascertained via a CCK-8 assay. With the rise in drug concentrations, the proliferation inhibitory rates of A2780 cells reached 49.0, 41.7 and $67.3 \%$ with single olaparib, cisplatin and paclitaxel from $14.0,10.0$ and $3.3 \%$, respectively, in a concentration-dependent manner. The proliferation inhibitory rates of OVCAR-3 cells reached 58.7, 70.1 and $70.7 \%$ with single olaparib, cisplatin and paclitaxel from $9.9,17.4$ and $18.0 \%$, respectively, in a concentration-dependent manner. It was noted that the inhibitory rates of two types of ovarian cell lines in the cisplatin + olaparib group were higher than those in any of the single group at all concentrations (Fig. 1B and D).

Synergistic, additive and antagonistic effects of combined agents on the proliferation inhibitory rate of $A 2780$ and OVCAR-3 cells. The data for the proliferation inhibitory rates of the three agents in single or combination use at 6 concentrations $(0.0625 \mathrm{x}, 0.125 \mathrm{x}, 0.25 \mathrm{x}, 0.5 \mathrm{x}, 1.0 \mathrm{x}$ and $2.0 \mathrm{x} \mathrm{IC}_{50}$ ) were substituted into Compusyn software, and the Compusyn provided graphic representations. The association between dose and effect was ascertained in accordance with the median-effect principle (Fig. 2A) (23). The synergistic, additive or antagonistic effects on proliferation inhibitory rates of the cells were dependent of the CI values. As a result, for the two ovarian cell lines, the cisplatin + olaparib group exhibited lower CI values than the other two combination groups at all concentrations. For A2780 cells, the CI value of cisplatin + olaparib group was upregulated to 1.14 from 0.32 across $\mathrm{EC}_{50}$ to $\mathrm{EC}_{75}$ (Table I). Cisplatin + olaparib with low concentrations $(0.0625 \mathrm{x}$, $0.125 \mathrm{x}$ and $0.25 \mathrm{x} \mathrm{C}_{50}$ ) exerted a strong synergistic effect, with CI values ranging from 0.1-0.3 for the fraction affected by the dose $(\mathrm{Fa})$ from $33.3-50.7 \%$. Cisplatin + olaparib at high concentrations $\left(0.5 \mathrm{x}, 1.0 \mathrm{x}\right.$ and $\left.2.0 \mathrm{IC}_{50}\right)$ exhibited a synergistic effect with $\mathrm{CI}$ values from 0.3-0.7, and $\mathrm{Fa}$ values from 50.3-63.7\%. For OVCAR-3 cells, cisplatin + olaparib at low concentrations $\left(0.0625 \mathrm{x}, 0.125 \mathrm{x}\right.$ and $\left.0.25 \mathrm{x} \mathrm{IC}_{50}\right)$ exerted a synergistic effect, with CI values ranging from 0.35-0.87, and $\mathrm{Fa}$ values from $32.4-39.9 \%$ (Fig. 2B). As the Fa values were $0.25,0.40$ and 0.55 , the cisplatin + olaparib combination demonstrated a stronger synergistic effect than the other two combinations. For OVCAR-3 cells, the Fa values were 0.25 and 0.40 , and thus the cisplatin + olaparib combination demonstrated stronger synergistic effects than the other two combinations (Fig. 2C).

Furthermore, for the two types of ovarian cell lines, the cisplatin + olaparib group at all concentrations $(0.0625 \mathrm{x}$, $0.125 \mathrm{x}, 0.25 \mathrm{x}, 0.5 \mathrm{x}, 1.0 \mathrm{x}$ and $\left.2.0 \mathrm{x} \mathrm{IC}_{50}\right)$ exhibit a higher dose-reduction index $>1$ than the other two combinations. For A2780 cells, single cisplatin and olaparib displayed 26.11and 12.73 -fold dose reductions at $0.125 \mathrm{x} \mathrm{IC}_{50}$ (Table II). It was suggested that, in the A2780 cell line, the CI values between the low concentrations $(0.0625 \mathrm{x}, 0.125 \mathrm{x}$ and $0.25 \mathrm{x}$ $\left.\mathrm{IC}_{50}\right)$ and high concentrations $\left(0.5 \mathrm{x}, 1.0 \mathrm{x}\right.$ and $\left.2.0 \mathrm{x} \mathrm{IC}_{50}\right)$ of cisplatin + olaparib were significantly different $(\mathrm{P}<0.05$; Fig. 3A). In the OVCAR-3 cell line, the CI values of low concentrations $\left(0.0625 \mathrm{x}, 0.125 \mathrm{x}\right.$ and $\left.0.25 \mathrm{x} \mathrm{IC}_{50}\right)$ presented synergistic effects (Fig. 3B).

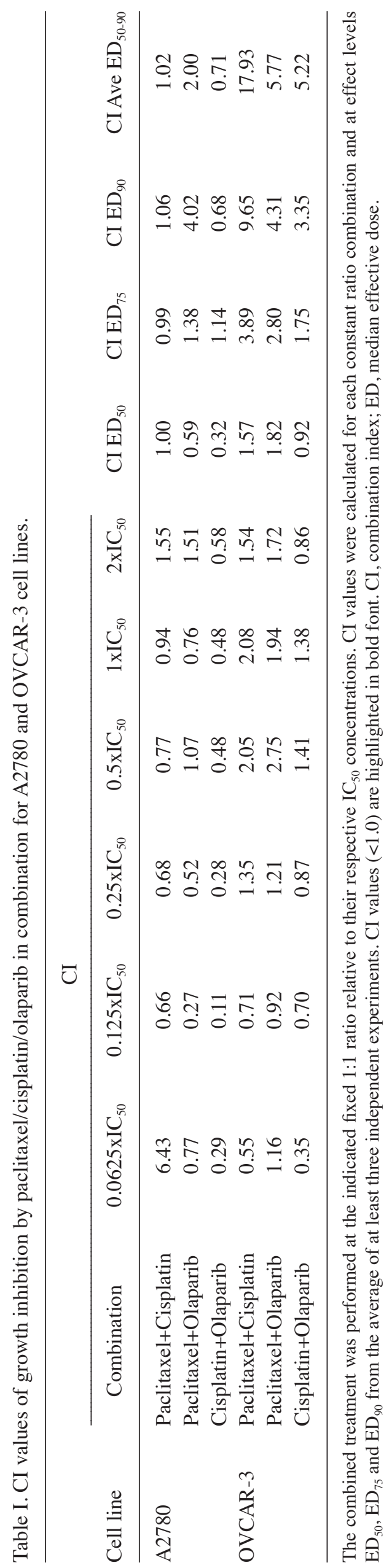


Table II. DRI values for growth inhibition by paclitaxel/cisplatin/olaparib in combination for A2780 and OVCAR-3 cell lines.

\begin{tabular}{|c|c|c|c|c|c|c|c|c|}
\hline \multirow[b]{2}{*}{ Cell line } & \multirow[b]{2}{*}{ Combination } & \multirow[b]{2}{*}{ Component } & \multicolumn{6}{|c|}{ DRI } \\
\hline & & & $0.0625 \times \mathrm{xIC}_{50}$ & $0.125 \times \mathrm{IC}_{50}$ & $0.25 \mathrm{xIC}_{50}$ & $0.5 \times \mathrm{IC}_{50}$ & $1 \times \mathrm{XC}_{50}$ & $2 \mathrm{xIC}_{50}$ \\
\hline \multirow[t]{6}{*}{ A2780 } & \multirow[t]{2}{*}{ Paclitaxel+Cisplatin } & Paclitaxel & 0.75 & 2.29 & 1.89 & 1.53 & 1.17 & 0.70 \\
\hline & & Cisplatin & 0.20 & 4.49 & 6.50 & 8.97 & 11.12 & 8.04 \\
\hline & \multirow[t]{2}{*}{ Paclitaxel+Olaparib } & Paclitaxel & 4.09 & 5.26 & 2.69 & 1.32 & 1.46 & 0.73 \\
\hline & & Olaparib & 1.89 & 12.73 & 6.74 & 3.18 & 14.15 & 7.07 \\
\hline & \multirow{2}{*}{ Cisplatin+Olaparib } & Cisplatin & 13.83 & 26.11 & 11.27 & 6.34 & 5.74 & 4.36 \\
\hline & & Olaparib & 4.54 & 12.73 & 5.26 & 3.06 & 3.31 & 2.84 \\
\hline \multirow[t]{6}{*}{ OVCAR-3 } & \multirow[t]{2}{*}{ Paclitaxel+Cisplatin } & Paclitaxel & 3.30 & 2.60 & 1.37 & 0.90 & 0.91 & 1.27 \\
\hline & & Cisplatin & 4.07 & 3.11 & 1.63 & 1.06 & 1.02 & 1.33 \\
\hline & \multirow[t]{2}{*}{ Paclitaxel+Olaparib } & Paclitaxel & 1.51 & 1.91 & 1.45 & 0.64 & 0.90 & 1.01 \\
\hline & & Olaparib & 2.00 & 2.54 & 1.94 & 0.85 & 1.21 & 1.37 \\
\hline & \multirow[t]{2}{*}{ Cisplatin+Olaparib } & Cisplatin & 5.45 & 2.72 & 2.14 & 1.32 & 1.32 & 2.03 \\
\hline & & Olaparib & 6.02 & 3.01 & 2.45 & 1.53 & 1.62 & 2.72 \\
\hline
\end{tabular}

The combined treatment was performed at the indicated fixed 1:1 ratio relative to their respective $\mathrm{IC}_{50}$ concentrations. DRI values were calculated from the DRI equation and algorithm using CompuSyn software. Favorable DRI values $(>1.0)$ are highlighted in bold font. DRI, dose reduction index.

Combinational interactions of cisplatin and olaparib against the proliferation of A2780 and OVCAR-3 cells at low doses. The experiment was divided into four groups, cisplatin, olaparib, cisplatin + olaparib, and control group. The proliferation inhibitory effect of A2780 and OVCAR-3 cells was visually observed following crystal violet solution staining. Compared with the control group, the experimental groups (cisplatin, olaparib, cisplatin + olaparib) at $0.25 \mathrm{x} \mathrm{IC}_{50}$ all exhibited different levels of proliferation inhibition (Fig. 4A and C). The combination group exhibited the minimum crystal violet and the lowest fraction area of stained cells, as analyzed by ImageJ software, in each dish, revealing that the proliferation inhibitory effect was the strongest among four groups $(\mathrm{P}<0.01 ; \mathrm{Fig}$. $4 \mathrm{~B}$ and $\mathrm{D})$. In addition, the potent synergistic effects of cisplatin + olaparib were further revealed through cell apoptosis and SP cell via flow cytometry in A2780 and OVCAR-3 (Fig. S1). When the concentrations of cisplatin and olaparib were at $0.25 \mathrm{x} \mathrm{IC}$, the apoptotic rate in A2780 cells in the control, cisplatin, olaparib, cisplatin + paclitaxel, paclitaxel + olaparib, cisplatin + olaparib groups reached 2.08, 3.95, 6.40,7.28, 8.49 and $21.94 \%$, respectively. The apoptotic rate in OVCAR-3 cells in the control, cisplatin, olaparib, cisplatin + paclitaxel, paclitaxel + olaparib, cisplatin + olaparib groups reached 3.01, $5.24,8.74,9.78,12.25$ and $19.42 \%$, respectively (Fig. 4E and G). It was therefore demonstrated that combination group of cisplatin and olaparib at low doses can significantly induce the apoptosis of A2780 and OVCAR-3 cells compared with each single group and control group $(\mathrm{P}<0.01$; Fig. $4 \mathrm{~F}$ and $\mathrm{H})$. Besides, through sorting the side-population cells in A2780 and OVCAR-3, the percentage of SP cells showed quite different in the six groups. Following cisplatin, olaparib, cisplatin + paclitaxel, paclitaxel + olaparib, cisplatin + olaparib treatment, SP proportion in A2780 was respectively $8.11,8.02,7.47,5.91$ and $2.42 \%$, SP proportion in OVCAR-3 was respectively 8.05, 7.93, 6.21, 5.99 and $2.38 \%$. Cisplatin + olaparib can obviously decrease the proportion of SP cells (Fig. S1).

\section{Discussion}

Overall, combination chemotherapeutic agents exhibit improved efficacy than single administration for the majority of malignancies. The additive or synergistic effects of multiple agents usually lead to noticeably higher clinical benefit of the agents. Researchers have achieved breakthroughs in high-throughput sequencing and molecular targeted drugs, patients with ovarian cancer greatly benefit from these advanced technologies. Molecular-targeted drugs indicate that small molecule drugs primarily target critical sites to interfere with the pathogenesis and development of malignancies in a pathophysiological manner. The targeted drugs can not only be utilized in combination with chemotherapy, but can also act as maintenance therapy to promote patient survival time. At present, olaparib has exhibited encouraging therapeutic effects for the treatment of ovarian cancer. Olaparib combined with chemotherapy has been proven to enhance the efficacy of chemotherapy in patients with platinum-sensitive ROC. From the experimental results of the present study, it has been revealed that cisplatin + olaparib was the only group with a $\mathrm{CI}<0.7$ under all $\mathrm{Fa}$ values, revealing that the combination exerts synergistic or strong synergistic effects. Furthermore, the CI value was smaller than those of the other two combinations for each experimental concentration, which demonstrated its synergistic effect was superior.

Conventional anti-tumor therapies (radiotherapy and chemotherapy) exhibit favorable short-term efficacy largely by decimating tumor cells, resulting in fast shrinking of tumor lesions. Nevertheless, these methods are always accompanied by metastatic or recurrent tumors, which frustrate former efforts of the conventional strategies. Therefore, the combined use of PARP inhibitors can enhance the efficacy of radiotherapy, alkylation agents and platinum drug chemotherapy by inhibiting DNA damage repair of tumor cells and accelerating 


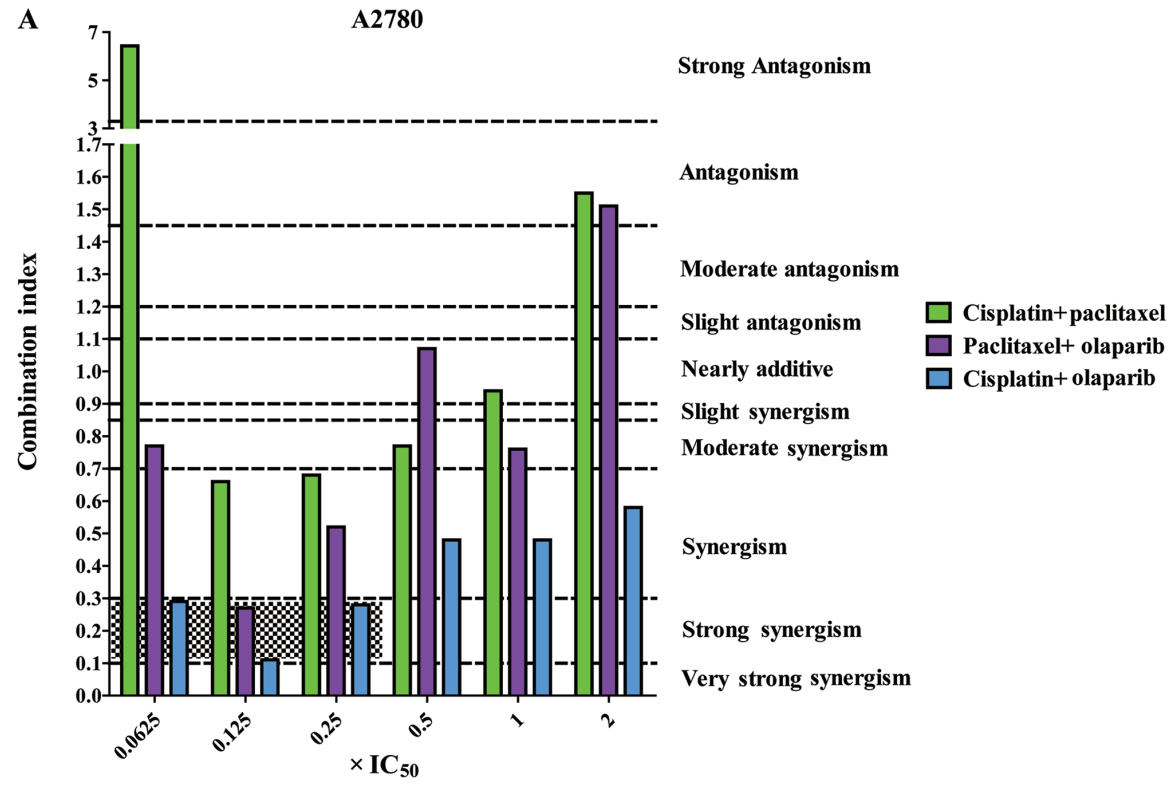

B

OVCAR-3

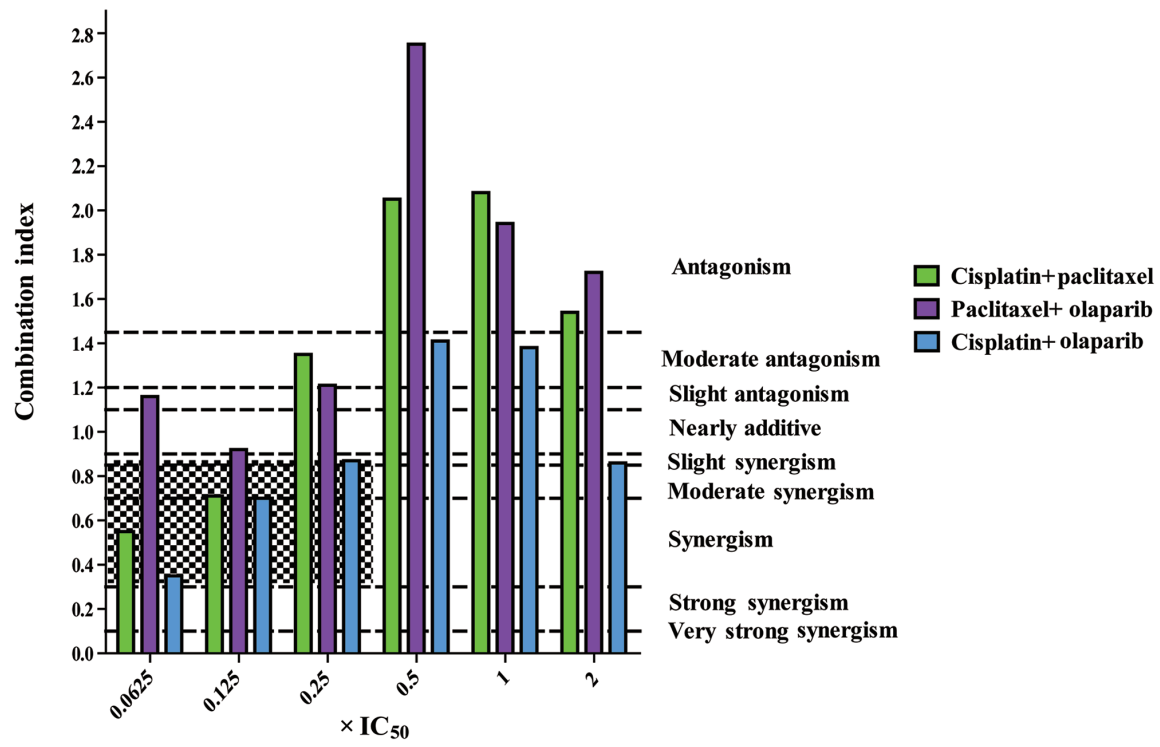

Figure 3. CI values of the combination of three agents in pairs at six concentrations $\left(0.0625,0.125,0.25,0.5,1\right.$ and $\left.2 x \mathrm{IC}_{50}\right)$. (A) $\mathrm{CI}$ values of the combinations in A2780 cells. (B) CI values of the combinations in OVCAR-3 cells. Range of CI: $<0.1$, very strong synergism; $0.1-0.3$, strong synergism; $0.3-0.7$, synergism; 0.7-0.85, moderate synergism; $0.85-0.90$, slight synergism; $0.90-1.10$, nearly additive; $1.10-1.20$, slight antagonism; $1.20-1.45$, moderate antagonism; $1.45-3.3$, antagonism; $3.3-10$, strong antagonism; $>10$, very strong antagonism. CI, combination index.

apoptosis of tumor cells (24). Adverse reactions can also be inhibited by decreasing chemoradiotherapy or radiation doses. It has been proved that low-dose chemotherapy drugs do not affect prognosis, and they can also significantly decrease the incidence of postoperative adverse reactions (25). As reported by Garcia and Singh (26) bevacizumab, in combination with low-dose chemotherapeutic agents, decreases the recurrent ovarian tumor recurrence and inhibits the growth of the lesion. The graphic representations and quantification outcomes in the present study demonstrate that the combination of cisplatin and olaparib exerts superior synergistic effects on the inhibition of A2780 and OVCAR-3 cell lines proliferation, particularly at low doses. The low-dose group $(0.0625 \mathrm{x}, 0.125 \mathrm{x}$ and $0.25 \mathrm{x}$ $\mathrm{IC}_{50}$ ) of cisplatin + olaparib even could reach the same proliferation inhibitory rate as high-dose group $(0.5 \mathrm{x}, 1.0 \mathrm{x}$ and $2.0 \mathrm{x}$ $\left.\mathrm{IC}_{50}\right)$ in the A2780 cells ( $\left.\mathrm{P}>0.05\right)$. Chemotherapy-induced cell cycle arrest is commonly considered a result of DNA damage. When DNA damage repair induced by chemotherapy cannot be achieved, cell aging or even apoptosis will be immediately initiated (27). In the present study, cisplatin + olaparib at low concentrations successfully induced the apoptosis of A2780 and OVCAR-3 ovarian cancer cell lines, exhibiting higher apoptosis rates than either single application. The combination significantly inhibited cell proliferation following apoptosis.

Furthermore, many studies have confirmed that cisplatin combined with olaparib has a synergistic effect on tumors in vivo. Minami et al (28) researched the effectiveness of the cisplatin with olaparib in a PTEN-deficient lung cancer xenograft model, they found that cisplatin plus olaparib could inhibit tumor growth than other treatment groups in PC-9 ${ }^{\text {PTEN }}$ xenograft model. Yasukawa et al (29) examined the effects of PARP inhibitor (AZD2281) with cisplatin on oral 
A

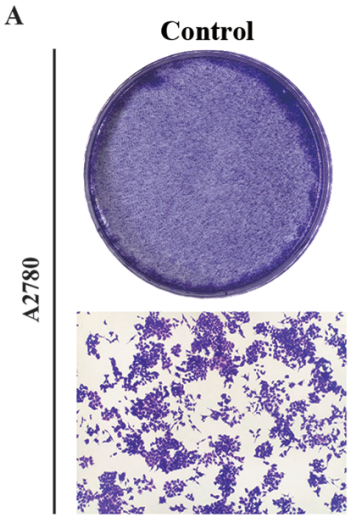

C

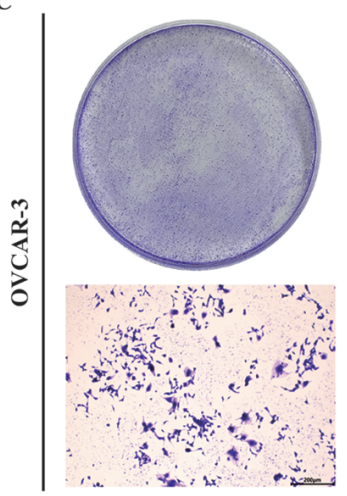

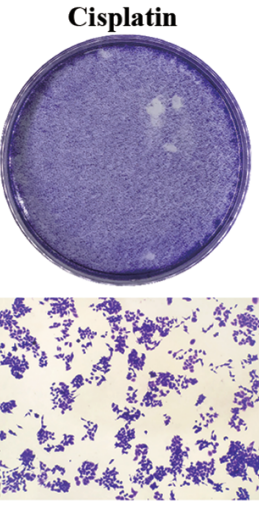
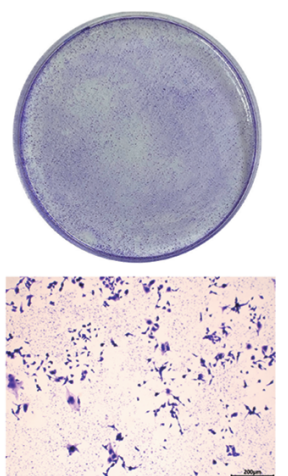
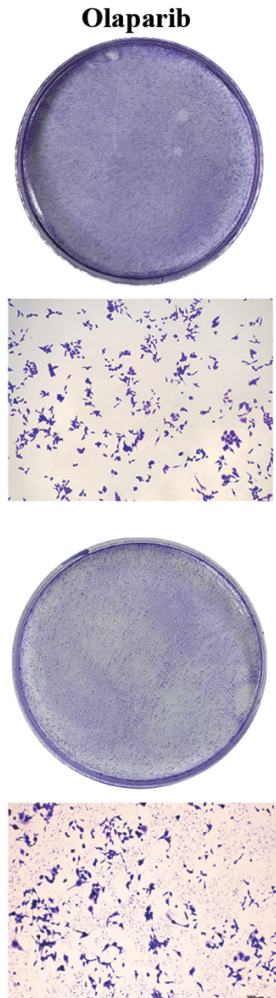
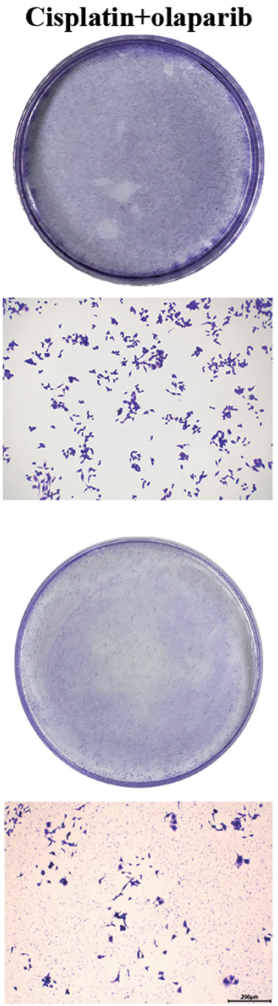

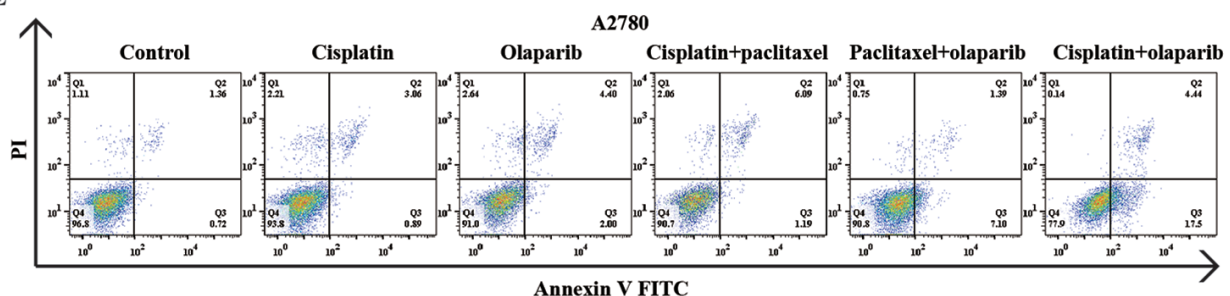

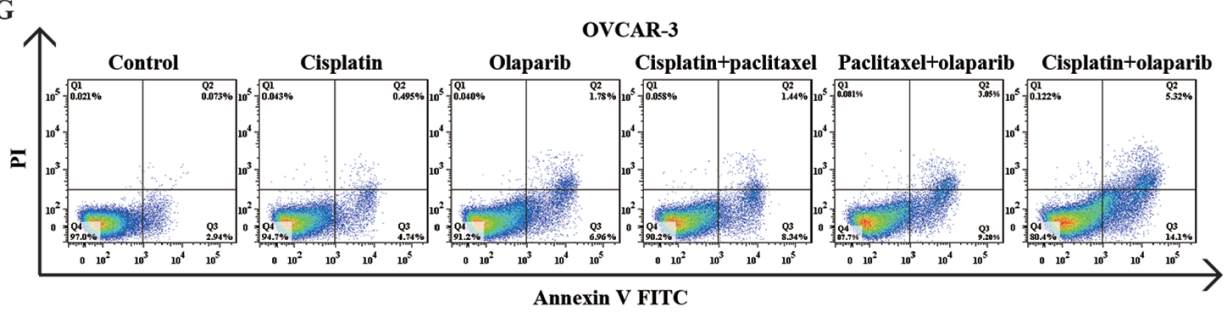

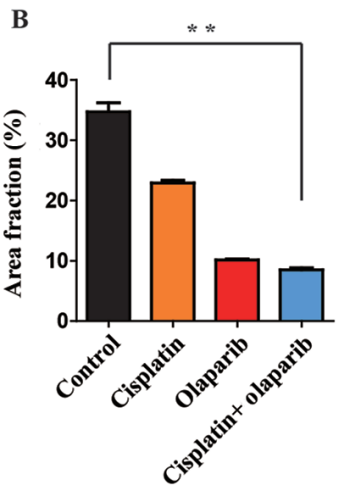

D
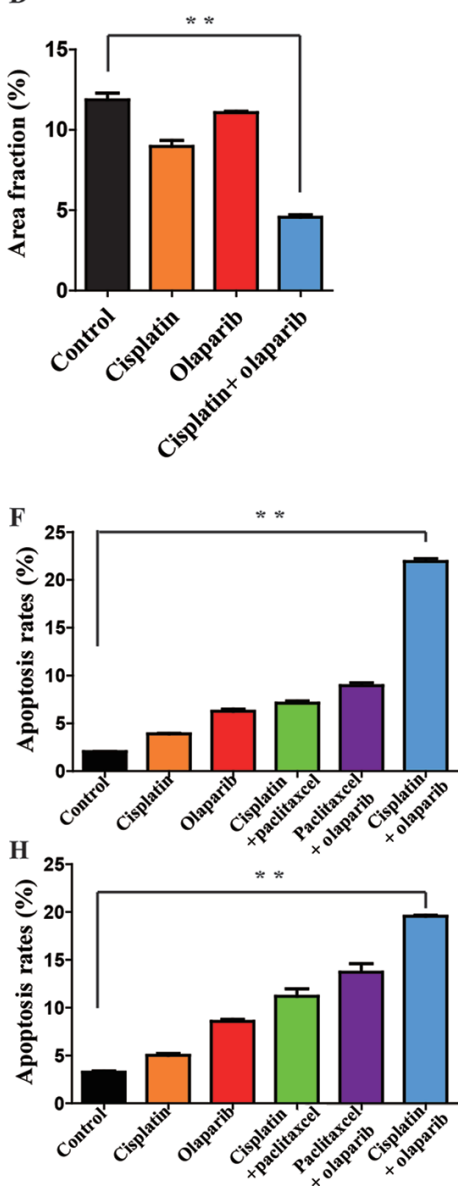

Figure 4. Effect of low-dose treatments on the proliferation of ovarian cancer cells. (A) Role of four groups in the proliferation of A2780 cells examined by a crystal violet assay. The $60-\mathrm{mm}$ cell culture dishes were observed and imaged under a microscope (magnification, x100). (B) Rate of stained cells was determined using ImageJ software. Data are presented as the mean \pm SEM ( $n=3$ ). (C) Role of four groups in the proliferation of OVCAR-3 cells examined by a crystal violet assay. (D) Rate of stained cells was determined using ImageJ software. Data are presented as the mean \pm SEM (n=3). (E) Role of six groups in the apoptosis of A2780 cells assessed by flow cytometry. (F) Rate of apoptosis was determined using FlowJo 7.6.1 software. (G) Role of six groups in the apoptosis of OVCAR-3 cells assessed by flow cytometry. (H) Rate of apoptosis was determined using FlowJo 7.6.1 software. ${ }^{* *} \mathrm{P}<0.01$.

cancer xenografted model. Results showed that combination treatment with AZD2281 and cisplatin significantly inhibited xenografted tumor growth compared with control and single treatment. de Groot et al (30) demonstrated that combined cisplatin and PARP1 inhibition could successfully attenuated tumor onset in a mouse model of BRCA1-associated breast cancer. Nevertheless, further studies are needed to elucidate the mechanisms behind the synergistic effect of olaparib combine with cisplatin on ovarian cancer cells in vivo and in vitro.

\section{Acknowledgements}

Not applicable.

\section{Funding}

The present study was supported by funding from National Key R\&D Program of China (grant no. 2016YFC1303100) and National Natural Science Foundation of China (grant nos. 31570803, 81773090, 81272879 and 81402151). 


\section{Availability of data and materials}

All data generated or analyzed during this study are included in this published article.

\section{Authors' contributions}

JG analyzed the growth inhibitory effects of single and combined anticancer agents on A2780 and OVCAR-3 cells and interpreted the CompuSyn report. ZW performed the experiments in cellular function and was a major contributor in writing the manuscript. JF and JA performed data acquisition. YO was involved in the conceptualization and investigation. CX conceived the study and contributed to review/editing of the manuscript. All authors read and approved the final manuscript.

\section{Ethics approval and consent to participate}

Not applicable.

\section{Patient consent for publication}

Not applicable.

\section{Competing interests}

The authors declare that they have no competing interests.

\section{References}

1. Siegel R, Ma J, Zou Z and Jemal A: Cancer statistics, 2014. CA Cancer J Clin 64: 9-29, 2014.

2. Bacalbasa N, Balescu I, Dima S, Brasoveanu V and Popescu I: Hematogenous splenic metastases as an independent negative prognosis factor at the moment of primary cytoreduction in advanced stage epithelial ovarian cancer-a single center experience. Anticancer Res 35: 5649-5654, 2015.

3. Rosell R, Lord RV, Taron M and Reguart N: DNA repair and cisplatin resistance in non-small-cell lung cancer. Lung Cancer 38: 217-227, 2002.

4. Michailidou M, Brown HK, Lefley DV, Evans A, Cross SS, Coleman RE, Brown NJ and Holen I: Microvascular endothelial cell responses in vitro and in vivo: Modulation by zoledronic acid and paclitaxel? J Vasc Res 47: 481-493, 2010.

5. Scholz HS, Tasdemir H, Hunlich T, Turnwald W, Both A and Egger H: Multivisceral cytoreductive surgery in FIGO stages IIIC and IV epithelial ovarian cancer: Results and 5-year follow-up. Gynecol Oncol 106: 591-595, 2007.

6. Vida A, Márton J, Mikó E and Bai P: Metabolic roles of poly(ADP-ribose) polymerases. Semin Cell Dev Bio 65: 135-143, 2017.

7. Schreiber V, Dantzer F, Ame JC and de Murcia G: Poly(ADP-ribose): Novel functions for an old molecule. Nat Rev Mo Cell Bio 7: 517-528, 2006.

8. Walsh CS: Two decades beyond BRCA1/2: Homologous recombination, hereditary cancer risk and a target for ovarian cancer therapy. Gynecol Oncol 137: 343-350, 2015.

9. He JX, Wang M, Huan XJ, Chen CH, Song SS, Wang YQ, Liao XM, Tan C, He Q, Tong LJ, et al: Novel PARP1/2 inhibitor mefuparib hydrochloride elicits potent in vitro and in vivo anticancer activity, characteristic of high tissue distribution. Oncotarget 8: 4156-4168, 2017.

10. Phulwani NK and Kielian T: Poly (ADP-ribose) polymerases (PARPs) 1-3 regulate astrocyte activation. J Neurochem 106: 578-590, 2008

11. Kummar S, Chen A, Parchment RE, Kinders RJ, Ji J, Tomaszewski JE and Doroshow JH: Advances in using PARP inhibitors to treat cancer. BMC Med 10: 25, 2012.

12. Ricks TK, Chiu HJ, Ison G, Kim G, McKee AE, Kluetz P and Pazdur R: Successes and challenges of PARP inhibitors in cancer therapy. Front Oncol 5: 222, 2015.
13. Löser DA, Shibata A, Shibata AK, Woodbine LJ, Jeggo PA and Chalmers AJ: Sensitization to radiation and alkylating agents by inhibitors of poly(ADP-ribose) polymerase is enhanced in cells deficient in DNA double-strand break repair. Mol Cancer Ther 9: 1775-1787, 2010.

14. Dedes KJ, Wilkerson PM, Wetterskog D, Weigelt B, Ashworth A and Reis-Filho JS: Synthetic lethality of PARP inhibition in cancers lacking BRCA1 and BRCA2 mutations. Cell Cycle 10: 1192-1199, 2011.

15. Ledermann J, Harter P, Gourley C, Friedlander M, Vergote I, Rustin G, Scott CL, Meier W, Shapira-Frommer R, Safra T, et al: Olaparib maintenance therapy in patients with platinum-sensitive relapsed serous ovarian cancer: A preplanned retrospective analysis of outcomes by BRCA status in a randomised phase 2 trial. Lancet Oncol 15: 852-861, 2014.

16. Parkes EE and Kennedy RD: Clinical application of Poly(ADP-ribose) polymerase inhibitors in high-grade serous ovarian cancer. Oncologist 21: 568-593, 2016.

17. Qin C, Zhang C, Zhu F, Xu F, Chen SY, Zhang P, Li YH, Yang SY, Wei YQ, Tao L and Chen YZ: Therapeutic target database update 2014: A resource for targeted therapeutics. Nucleic Acids Res 42 (Database Issue): D1118-D1123, 2014.

18. Fong PC, Yap TA, Boss DS, Carden CP, Mergui-Roelvink M, Gourley C, De Greve J, Lubinski J, Shanley S, Messiou C, et al: Poly(ADP)-ribose polymerase inhibition: Frequent durable responses in BRCA carrier ovarian cancer correlating with platinum-free interval. J Clin Oncol 28: 2512-2519, 2010.

19. Lee JM, Hays JL, Annunziata CM, Noonan AM, Minasian L, Zujewski JA, Yu M, Gordon N, Ji J, Sissung TM, et al: Phase I/Ib study of olaparib and carboplatin in BRCA1 or BRCA2 mutation-associated breast or ovarian cancer with biomarker analyses. J Natl Cancer Inst 106: dju089, 2014.

20. Del Conte G, Sessa C, von Moos R, Viganò L, Digena T, Locatelli A, Gallerani E, Fasolo A, Tessari A, Cathomas R and Gianni L: Phase I study of olaparib in combination with liposomal doxorubicin in patients with advanced solid tumours. Br J Cancer 111: 651-659, 2014.

21. Konstantinopoulos PA, Ceccaldi R, Shapiro GI and D'Andrea AD: Homologous recombination deficiency: Exploiting the fundamental vulnerability of ovarian cancer. Cancer Discov 5: 1137-1154, 2015.

22. Chou TC and Martin N: CompuSyn for Drug Combinations: PC Software and User's Guide: A computer program for quantitation of synergism and antagonism in drug combinations, and the determination of $\mathrm{IC}_{50}$ and $\mathrm{ED}_{50}$ and $\mathrm{LD}_{50}$ values. ComboSyn Inc., Paramus, NJ, 2005.

23. Ashton JC: Drug combination studies and their synergy quantification using the Chou-Talalay method-letter. Cancer Res 75: 2400, 2015.

24. Oza AM, Cibula D, Benzaquen AO, Poole C, Mathijssen RH, Sonke GS, Colombo N, Špaček J, Vuylsteke P, Hirte H, et al: Olaparib combined with chemotherapy for recurrent platinum-sensitive ovarian cancer: A randomised phase 2 trial. Lancet Oncol 16: 87-97, 2015.

25. Kamada K, Nakanishi T, Kitamoto M, Aikata H, Kawakami Y, Ito K, Asahara T and Kajiyama G: Long-term prognosis of patients undergoing transcatheter arterial chemoembolization for unresectable hepatocellular carcinoma: Comparison of cisplatin lipiodol suspension and doxorubicin hydrochloride emulsion. J Vasc Interv Radiol 12: 847-854, 2001.

26. Garcia A and Singh H: Bevacizumab and ovarian cancer. Ther Adv Med Oncol 5: 133-141, 2013.

27. Salminen A, Ojala J and Kaarniranta K: Apoptosis and aging: Increased resistance to apoptosis enhances the aging process. Cell Mol Life Sci 68: 1021-1031, 2011.

28. Minami D, Takigawa N, Takeda H, Takata M, Ochi N, Ichihara E, Hisamoto A,Hotta K, Tanimoto M and Kiura K: Synergistic effect of olaparib with combination of cisplatin on PTEN-deficient lung cancer cells. Mol Cancer Res 11: 140-148, 2013.

29. Yasukawa M, Fujihara H, Fujimori H, Kawaguchi K, Yamada H, Nakayama R, Yamamoto N, Kishi Y, Hamada Y and Masutani M: Synergetic effects of PARP inhibitor AZD2281 and cisplatin in oral squamous cell carcinoma in vitro and in vivo. Int J Mol Sci 17: 272, 2016.

30. de Groot JS, van Diest PJ, van Amersfoort M, Vlug EJ, Pan X, Ter Hoeve ND, Rosing H, Beijnen JH, Youssef SA, de Bruin A, et al: Intraductal cisplatin treatment in a BRCA-associated breast cancer mouse model attenuates tumor development but leads to systemic tumors in aged female mice. Oncotarget 8: 60750-60763, 2017. 